\title{
Precisely reduced meteoroid trajectories and orbits from the 1995 Leonid meteor outburst
}

\author{
Hans Betlem, ${ }^{1}$ Casper ter Kuile, ${ }^{1}$ Jaap van't Leven, ${ }^{1}$ Marc de Lignie, ${ }^{1}$ Luis Ramon Bellot, ${ }^{2}$ Mike Koop, ${ }^{3}$ \\ Chris Angelo, ${ }^{3}$ Mike Wilson ${ }^{4}$ and Peter Jenniskens ${ }^{4}$ \\ 'Dutch Meteor Society, I ederkarper 4, NI -2318 NR I eiden. The Netherlands \\ ${ }^{2}$ Instituto de Astrofisica de Canaria, E-38200 La Laguna, Tenerife. Spain \\ ${ }^{3}$ Fremont Peak Observatory Association, P.O. Box 1110 . San Juan Batista, CA 95045, L.S.A. \\ ${ }^{4}$ NASA/Ames Research Center, M.S. 239-4, Moffett Field. CA 94035-1000, U.S.A.
}

Received 11 September 1996; accepted 9 December 1996

\begin{abstract}
The discovery of enhanced Leonid activity in 1994 led to the mounting of a multi-station photographic campaign on two continents during the Leonids of 1995. The goal was to determine orbits and trajectories of outburst Leonids. The outburst did recur as predicted, resulting in 23 precisely reduced Leonid orbits and 30 Leonid trajectories. Individual velocities and radiant positions, as well as the average orbit are presented. It is found that the distribution of Leonid radiants contains a dense cluster of seven radiant positions. This clustering is tentatively associated with the visually observed outburst component. These seven meteors provide the first orbital elements of outburst Leonids and put strong constraints on theoretical models of the origin and evolution of the Leonid stream. (C) 1997 Elsevier Science Ltd
\end{abstract}

\section{Introduction}

With the discovery of enhanced activity from the Leonid meteor shower in 1994 (Jenniskens, 1996), it became clear that the upcoming years already present a unique opportunity to study the dust distribution near the orbit of parent comet $\mathrm{P} /$ Tempel-Tuttle. This includes measuring the meteoroid stream activity profiles from year to year. as well as measuring the orbital elements of individual particles in the stream. The dust component for the present meteor outbursts may well differ from that responsible for the Leonid storms (Yeomans, 1981 ; Jenniskens. 1996; Yeomans e't al., 1996) and any information on the spatial distribution of dust will constrain numerical models on the formation and evolution of the Leonid meteor

Correspondence' to: H. Betlem' or P. Jenniskens stream (Wu and Williams, 1992. 1996: Brown and Jones. 1992). Most useful for that purpose are preciscly reduced orbits of individual particles.

Past observations of the I.eonid shower during the perihelion returns of 1932 and 1966 have been hampered severely by bad weather in November and by the restricted visibility of meteor outbursts on the globe. These considerations led us to organize a dedicated observation campaign for the 1995 Leonid return at two siles on different continents with a reasonable chance of clear weather. This work is part of an ongoing effort to study meteor outbursts, which are significant enhancements of meteor rates above the normal annual activity that are associated with relatively recent cometary ejecta (Jenniskens, 1995a).

Here we present the orbits and trajectories of 30 Leonid meteoroids that were measured during that campaign. These data provide the first precisely reduced orbital elements of Leonids that can be confidently associated with an outburst of the stream.

\section{The photographic network}

A four-station photographic network was employed in the Spanish province of Andalucia ( $4 \mathrm{~W} .+37 \mathrm{~N}$ ). Twenty trained visual observers of the Dutch Meteor Society DMS and seven observers of the Spanish Meteor Society SOMYCE provided the necessary records of time and location of bright meteors. A similar three-station network was employed in the San Francisco Bay Area of California $(122 \mathrm{~W},+37 \mathrm{~N})$. operated by ten amateur astronomers of the Fremont Peak Observatory Association and volunteers from NASA/Ames Research Center.

Based on accounts of the previous return of the comet in the 1960s (McIntosh and Millman. 1970), the I.eonid outburst of 1995 was expected to have a duration of close 
to a day and a peak rate of about 30 Leonids $\mathrm{h}^{-1}$, with a relatively high abundance of bright meteors (Jenniskens, 1996). The Leonid shower would be visible for a period of $6 \mathrm{~h}$ at each site, and both sites together would cover the interval of $23 \mathrm{~h}$ UT until $12 \mathrm{~h}$ UT. This allows a more complete coverage of the meteor stream profile, but also increases the possibility that the peak of the event would be recorded by one of the two stations. The networks were operated during the nights of November 16 and 17, while the European network was also operated during the nights of November 18-20.

The photographic data were reduced in much the same way as described in Betlem et al. (1997). A novelty was that all photographic multi-station images were transferred to Kodak Photo-CD. A measuring program was developed that enables reducing these digital photographic images with $3 \mu \mathrm{m}$ accuracy, resulting in the same overall accuracy as found from measurements on the negatives with the Jena Astrorecord measuring table (de Lignie, 1994, 1995). This program allows a much faster reduction of photographic data. The astrometrical data are then further reduced to derive orbital elements using the program FIRBAI (Ceplecha, 1987).

\section{Results}

The expedition was very successful. The Leonid stream peaked over Europe with a zenithal hourly rate of about 35 Leonids $h^{-1}, 2-3$ times the annual activity, and was rich in bright meteors (Brown, 1995; Jenniskens, 1995b). As a result, some 35 members of the Leonid stream were photographed from two or more sites, which enabled us to compute Leonid orbits of particles in the outburst component. Here, 30 precise trajectories and the average orbit are presented. A more complete analysis including multi-station intensified video observations of the Leonid stream will be published elsewhere.

Table 1 lists the appcarance timc of the Lconid meteors,

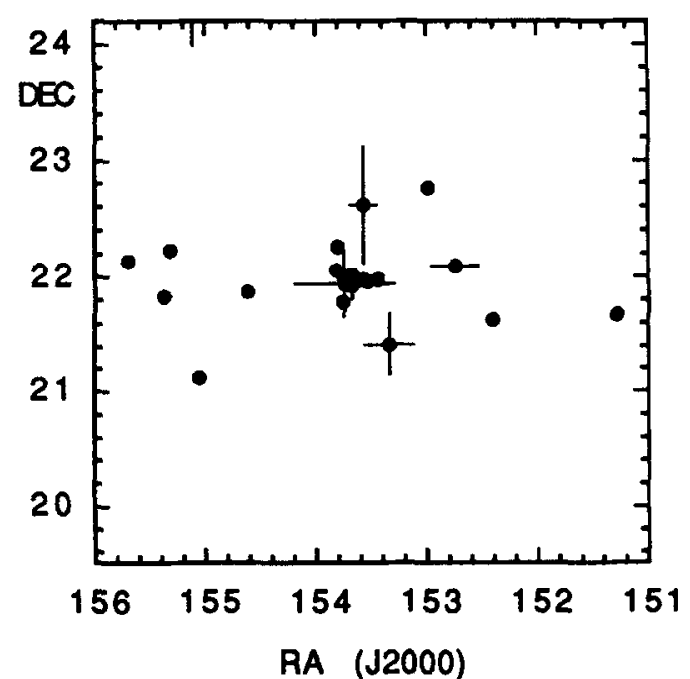

Fig. 1. Geocentric radiant positions, Leonids 1995 DMS. Radiant positions have been corrected for radiant drift to November $18,0 \mathrm{~h} \mathrm{UT}$ and radiants from trajectories with a $\mathrm{Q}$ angle smaller than $11^{\circ}$ have been removed. A radiant concentration at $\mathrm{RA}=253^{\circ} .63 ; \mathrm{DEC}=+21^{\circ} .97$ is obvious the visual magnitude, beginning and end height of the photographed meteor trajectory, geocentric velocity and radiant position, and the largest angles between any set of two planes fitted through meteor path and observing station. Images from the Spain network have serial numbers starting with " 952 ", while the results from the California network carry numbers starting with " 953 ". All radiant positions are given for equinox $\mathbf{J} 2000$.0. Error estimates are only given for meteors with three or more components.

Seven Leonids were too faint to obtain reliable values for the pre-atmospheric entry velocity $\left(V_{\text {inf }}\right)$ and for these meteors no orbital elements could be computed. However, the trajectory information results in an accurate radiant position even after allowing for a correction of zenith attraction with an adopted $V_{\text {inf }}$ of $72.0 \mathrm{~km} \mathrm{~s}^{-1}$.

The corrected geocentric radiant positions are shown in Fig. 1. Excluded are six solutions with a convergence angle of less than $11^{\circ}$. In order to show the intrinsic distribution of the direction vectors of the meteors, we allowed for a correction for daily drift of the radiant due to the changing orientation of the Earth's velocity vector. Hence, the positions were corrected for the radiant drift and normalized to November $18,0 \mathrm{~h} \mathrm{UT}$, using the daily motion in right ascension of $+0.99^{\circ}$ day $^{-1}$ and in declination $-0.36^{\circ}$ day $^{-1}$ (Jenniskens, 1988).

Seven of the 24 remaining radiant positions cluster around $\mathrm{RA}=153.63 \pm 0.11$ and $\mathrm{DEC}=+21.97 \pm 0.03$ (J2000.0). They are spread over the entire episode of outburst activity. $\Lambda$ part from this small subgroup, other Leonid radiants are dispersed within a few degrees, which is also typically found for annual Perseid meteors. There is no evidence for sub-radiants or structure in the dispersed component.

Orbital elements could be computed for 23 bright Leonids, which increases the number of known Leonid orbits by a factor of two. Table 2 lists the mean values of the orbital elements that follow from the data in Table 1. A distinction is made between the cluster of seven meteors and the other more dispersed meteors.

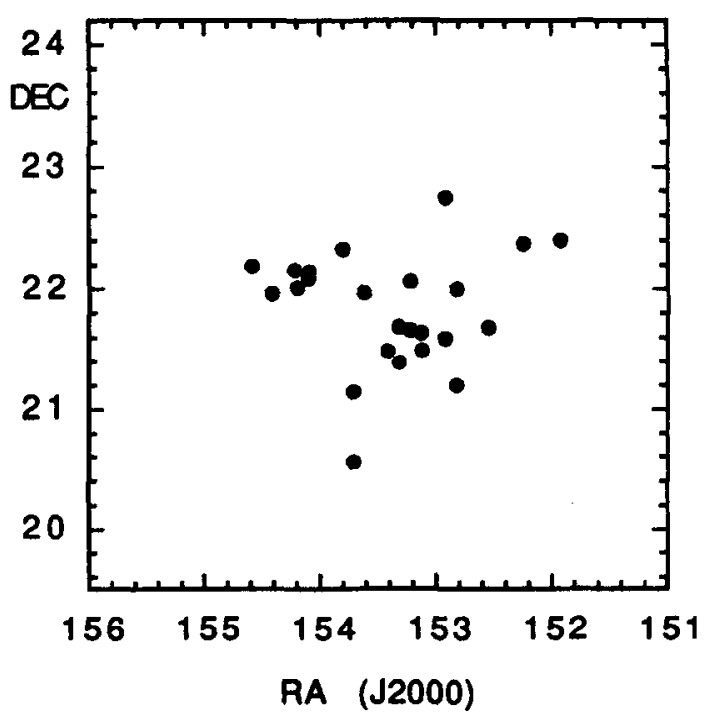

Fig. 2. Radiant positions of photographed Leonids in the IAU database $(N=24,1938-1980)$. Radiant positions are corrected for daily motion to the solar longitude of November 18,0 UT, 1995 and are for Eq. J2000.0 
Table 1. Thirty Leonid meteors photographed from two or more sites during the 1995 Leonid shower. The table lists the appearance time, absolute visual magnitude, beginning height $(\mathrm{km})$, end height $(\mathrm{km})$, geocentric velocity $\left(\mathrm{km} \mathrm{s}^{-1}\right)$, and geocentric radiation position (J2000), as well as the largest convergence angle of any set of two planes fitted through meteor path and station (degrees)

\begin{tabular}{|c|c|c|c|c|c|c|c|c|c|c|c|}
\hline Code & $\begin{array}{c}\text { Date } \\
\text { [Nov 95] }\end{array}$ & $M_{v}$ & H beg & $H$ end & V Geo & {$[+1-]$} & RA Geo & {$[+/-]$} & Dec Geo & {$[+1-1$} & $Q \max$ \\
\hline $95301^{*}$ & 17.3575 & 0 & 114.8 & 102.3 & 71.0 & 0.3 & 153.11 & & 22.22 & & 13.0 \\
\hline 95302 & 17.4000 & -2 & 112.5 & 98.9 & 71.3 & 1.4 & 1 53.57 & & 22.12 & & 6.5 \\
\hline 95303 & 17.4053 & -2 & 113.1 & 91.9 & 70.3 & 0.4 & 154.32 & 0.22 & 22.40 & 0.15 & 7.8 \\
\hline 95202 & 18.0120 & -3 & 117.5 & 90.4 & 71.0 & 0.3 & 155.12 & 0.52 & 24.75 & 0.76 & 2.1 \\
\hline $95207^{*}$ & 18.0436 & -3 & 114.1 & 94.4 & 70.4 & 0.5 & 153.72 & 0.05 & 22.00 & 0.01 & 34.2 \\
\hline 95212 & 18.0544 & -2 & 113.4 & 101.6 & 70.1 & 0.5 & 153.81 & 0.01 & 21.76 & 0.04 & 34.7 \\
\hline $95213^{*}$ & 18.0573 & -3 & 114.2 & 90.7 & 71.1 & 0.3 & 153.73 & 0.08 & 21.90 & 0.12 & 21.3 \\
\hline 95214 & 18.0573 & -1 & 113.7 & 95.1 & 70.4 & 0.2 & 153.39 & 0.22 & 21.39 & 0.27 & 34.8 \\
\hline 95216 & 18.0973 & -1 & 111.1 & 100.4 & 70.7 & 0.5 & 153.90 & & 22.22 & & 20.9 \\
\hline $95217^{*}$ & 18.0996 & 0 & 108.8 & 92.9 & 70.7 & 0.5 & 153.84 & 0.45 & 21.90 & 0.28 & 11.0 \\
\hline 95218 & 18.0109 & 0 & 107.0 & 99.7 & & & 154.89 & & 25.60 & & 11.5 \\
\hline 95219 & 18.1099 & 0 & 111.1 & 99.8 & 70.9 & 0.4 & 152.85 & 0.21 & 22.05 & 0.05 & 20.6 \\
\hline 95220 & 18.1174 & 0 & 107.3 & 94.9 & 70.7 & 0.7 & 154.04 & & 23.27 & & 10.9 \\
\hline $95221^{*}$ & 18.1215 & 0 & 108.7 & 95.5 & 71.1 & 0.4 & 153.56 & 0.03 & 21.93 & 0.04 & 65.3 \\
\hline 95222 & 18.1238 & -1 & 111.7 & 93.5 & 70.4 & 0.7 & 153.69 & 0.12 & 22.57 & 0.50 & 20.8 \\
\hline 95224 & 18.1253 & 0 & 107.4 & 87.0 & 71.8 & 0.8 & 153.11 & & 22.71 & & 19.4 \\
\hline 95225 & 18.1264 & 0 & 107.5 & 100.7 & & & 157.96 & & 22.31 & & 44.5 \\
\hline 95228 & 18.1332 & 0 & 110.8 & 97.7 & 71.1 & 1.2 & 157.93 & & 22.31 & & 26.0 \\
\hline 95229 & 18.1456 & 0 & 110.0 & 97.8 & & & 153.96 & & 22.00 & & 50.8 \\
\hline 95231 & 18.1542 & 0 & 108.7 & 94.1 & 71.7 & 0.9 & 155.47 & & 22.17 & & 21.4 \\
\hline 95232 & 18.1552 & 0 & 107.0 & 96.4 & 71.6 & 0.2 & 155.85 & 0.01 & 22.08 & 0.03 & 40.2 \\
\hline $95233^{*}$ & 18.1609 & -2 & 111.5 & 91.9 & 71.3 & 0.8 & 153.69 & 0.08 & 21.90 & 0.05 & 76.1 \\
\hline 95234 & 18.1689 & 0 & 110.3 & 93.8 & 71.1 & 2.4 & 155.54 & & 21.77 & & 36.5 \\
\hline 95235 & 18.1701 & 0 & 112.6 & 99.9 & & & 151.45 & & $21.6 !$ & & 25.1 \\
\hline 95236 & 18.1720 & 0 & 110.1 & 100.5 & & & 154.79 & & 21.81 & & 41.8 \\
\hline 95237 & 18.1763 & 0 & 109.0 & 98.6 & & & 155.23 & & 21.05 & & 17.8 \\
\hline $95238^{*}$ & 18.1788 & -2 & 113.1 & 94.0 & 70.8 & 1.5 & 153.75 & & 21.91 & & 61.9 \\
\hline 95312 & 18.4899 & -6 & 112.8 & 84.6 & 69.9 & 1.0 & 150.64 & & 20.91 & & 8.6 \\
\hline 95314 & 18.5611 & -1 & 108.6 & 94.2 & 69.8 & 1.5 & 155.14 & 1.20 & 21.84 & & 10.6 \\
\hline 95248 & 21.2403 & 0 & 115.1 & 99.5 & & & 155.63 & & 20.46 & & 50.9 \\
\hline Mean & & -1 & 111.1 & 95.8 & 70.8 & $(0.5)$ & 154.26 & $(0.12)$ & 22.16 & $(0.09)$ & 28.2 \\
\hline St. dev. & & & 2.8 & 4.3 & 0.5 & & 1.52 & & 0.98 & & \\
\hline
\end{tabular}

\section{Discussion}

The IAU photographic database (Lindblad, 1987, 1992) contains 24 Leonid meteor orbits from the period 19381980. The positions from the IAU database were transferred to 2000.0 coordinates and the same radiant drift correction was applied. The data are shown in Fig. 2. When comparing the orbital elements of the annual component with the IAU database mean values (Table 2), no significant differences are evident, which gives confidence in the reduction procedure.

Some of the Leonids in the IAU database were obtained in 1965 and 1966. It seems, however, that none of the meteors were obtained during the Leonid storm of 1966. A few meteors may have been part of the broad 1965 outburst. Again, we examined these data for a possible radiant clustering as we found from the 1995 data, but do not find a radiant as compact. We suspect that the given radiant positions are not precise enough to reveal the intrinsic dispersion in those past Leonid data.

It is unlikely that the large dispersion of radiants is indicative of high ejection velocities as proposed by $\mathrm{Wu}$ and Williams (1996). In fact, the small dispersion of the present orbital elements argues against a model with high

Table 2. Orbital elements of the two components of the 1995 Leonids stream : the annual stream and the outburst component. Results are compared with older data from the IAU Meteor database

\begin{tabular}{|c|c|c|c|c|c|c|c|c|}
\hline & & $q(\mathrm{AU})$ & $a^{-1}\left(\mathrm{AU}^{-1}\right)$ & $e$ & $i$ & $\omega$ & $\Omega$ & $\pi$ \\
\hline DMS 1995 & median & 0.983 & 0.067 & 0.933 & 161.6 & 171.9 & 235.27 & 47.2 \\
\hline$N=23(2000.0)$ & st. dev. & 0.005 & 0.057 & 0.057 & 1.5 & 3.9 & 0.29 & 4.0 \\
\hline DMS 1995 cluster & median & 0.984 & 0.068 & 0.933 & 162.2 & 172.6 & 235.22 & 47.8 \\
\hline$N=7(2000.0)$ & st. dev. & 0.000 & 0.027 & 0.027 & 0.1 & 0.4 & 0.29 & $\begin{array}{r}4.0 \\
0.6\end{array}$ \\
\hline IAU $1938-1980$ & median & 0.985 & 0.067 & 0.934 & 162.5 & 173.1 & 235.6 & 48.7 \\
\hline$N=24(2000.0)$ & st. dev. & 0.002 & 0.088 & 0.086 & 0.9 & 2.5 & 1.1 & 2.8 \\
\hline
\end{tabular}


ejection velocities. The model by $\mathrm{Wu}$ and Williams can, therefore, not be correct.

A cautionary note needs to be made, however. In all likelihood, the cluster of seven meteors is associated with the meteor outburst component. The small dispersion in radiant position is probably related to the smaller dispersion in the descending node of the outburst particles. The more dispersed radiant of all other meteors is then assumed to be due to the annual Leonid component. However, with a peak outburst rate of $32 \pm 5$, we would have expected to have photographed outburst versus annual Leonids in a ratio of $5: 3$. Hence, some of the more dispersed Leonids may well be part of the outburst component and the true dispersion may be somewhat larger than indicated.

\section{Conclusions}

We have provided orbital elements of the dust component that is responsible for the 1995 Leonid meteor outburst. The distribution of radiant positions shows a compact cluster. These are the first precisely reduced meteor orbits that can confidently be associated with any outburst of the Leonid stream. The result puts strong constraints on existing models of the Leonid meteor stream and argues for smaller ejection velocities than currently adopted.

Acknowledgements. These results were obtained as a result of the effort of a large group of amateur meteor observers. Each contributed invaluably by operating the camera batteries and by marking the time and sky position of the bright Leonids. Olga van Mil and Jeffrey Landlust did a major part of the time consuming data reduction. Financial and material support for the Andalucia expedition came from the Leidsche Kerkhoven Bosschafonds, Prins Bernhardfonds, Stichting Physica, Shell Nederland BV, Honda Power Equipment, Kodak Nederland BV, Ilford Nederland, Canon Benelux, Delft Electronic Products, Sony Nederland BV and Opdenkelder Technology. Hans Betlem is guest observer at the Leiden Sterrewacht. Peter Jenniskens is supported by the NASA/Planetary Astronomy Program.

\section{References}

Betlem, H., de Lignie, M. C., ter Kuile, C., van't Leven, J., Jobse, K., Miskotte, K. and Jenniskens, P. (1997) Precision meteor orbits of the Dutch Meteor Society-photographic meteor survey (1981-1993). Astron. Astrophys. Suppl. Ser. (in press).

Brown, P. (1995) Another Leonid enhancement, Bulletin 7 of the International Leonid Watch. WGN 23, 196-198.

Brown, P. and Jones, J. (1992) Evolution of the Leonid meteor stream. In Meteoroids and their Parent Bodies, eds J. Stohl and I. P. Williams, pp. 57-60.

Ceplecha, Z. (1987) Geometric, dynamic, orbital and photometric data on meteoroids from photographic fireball networks. Bull. Astron. Inst. Czech. 38, 222-234.

Jenniskens, P. (1988) Visual Observers Handbook. Dutch Meteor Society, Leiden.

Jenniskens, P. (1995a) Meteor stream activity. II. Meteor outbursts. Astron. Astrophys. 295, 206-235.

Jenniskens, P. (1995b) A second Leonid outburst in 1995. WGN 23, 198-200.

Jenniskens, P. (1996) Meteor stream activity. III. Measurement of the first in a new series of Leonid outbursts. Meteoritics Planet. Sci. 31, 177-184.

de Lignie, M. C. (1994) Measuring meteor negatives via Photo CD : a small revolution. Radiant 16, 5-12.

de Lignie, M. C. (1995) Astro Record 2.0. Radiant 17, 61-64.

Lindblad, B. A. (1987) Interplanetary Matter, Proc. 10th European Reg. Meeting of the $I A U$, eds Z. Ceplecha and P. Pecina, Vol. 2, pp. 201-204.

Lindblad, B. A. (1992) The orbit and mean radiant motion of the Leonid meteor stream. In Meteoroids and their Parent Bodies, eds I. Stohl and I. P. Williams, pp. 177-180.

McIntosh, B. A. and Millman, P. M. (1970) The Leonids by radar-1957-1968. Meteoritics 5, 1-18.

$\mathrm{Wu}, \mathrm{Z}$. and Williams, I. P. (1992) Formation of the Leonid meteor stream and storm. In Asteroids, Comets, Meteors, eds A. W. Harris and E. Bowell, pp. 661-665. LPI Houston, Texas.

Wu, Z. and Williams, I. P. (1996) Leonid meteor storms. Mon. Not. R. Astron. Soc. 280, 1210-1218.

Yeomans, D. K. (1981) Comet Tempel-Tuttle and the Leonid meteors. Icarus 47, 492-499.

Yeomans, D. K., Yau, K. K. and Weissman, P. R. (1996) The impending appearance of comet Tempel-Tuttle and the Leonid meteors. Icarus 124, 407-413. 